\title{
Uso de Proveniência de Objetos de Aprendizagem para Identificação do Estilo Preferencial de Aprendizagem
}

\author{
Thiago Nery, Gislaine Coelho, Fernanda Campos, Regina Braga, \\ Victor Stroële, José Maria N. David \\ Universidade Federal de Juiz de Fora \\ Programa de Pós-graduação em Ciência da Computação \\ Rua José Lourenço Kelmer, s/n-Campus Universitário- 36036-900-Juiz de Fora/MG \\ thiago.nery, fernanda.campos, regina.braga, jose.david \{@ufjf.edu.br\}; \\ victor.stroeledice.ufje.br, gislainemartins1990@gmail.com
}

\begin{abstract}
Data provenance allows capturing the historical documentation of learning objects (LO), both through their metadata and life trajectory. This article describes BROAD-ProvCapture architecture that is able to identify the student's main learning style, in a given educational situation, where the teacher previously selects the learning objects, capturing the sequence in a study section and using ontological rules to infer the features. This implicitly captured profile is incorporated into the recommendation system. Preliminary results point to the feasibility of the project.
\end{abstract}

Resumo. A proveniência de dados permite capturar a documentação histórica dos objetos de aprendizagem (OA), tanto através de seus metadados quanto de sua trajetória de vida. Esse artigo descreve a BROAD-ProvCapture, uma arquitetura capaz de identificar o estilo preferencial de aprendizagem do aluno, numa dada situação de ensino em que o professor seleciona previamente os objetos, capturando a sua seqüência da sessão de estudo e utilizando regras ontológicas para inferir as características. Esse perfil capturado implicitamente é incorporado ao sistema de recomendação. Os resultados preliminares apontam para a viabilidade do projeto.

\section{Introduçãa}

Na medida em que os Sistemas de Recomendação (SR) Educacionais buscam soluções mais aderentes ao perfil e contexto do aluno e dos grupos de alunos, cresce o interesse por recursos educacionais de qualidade. Esses recursos são descritos como objetos de aprendizagem (OA) [Valaski, 2011] e devem prover mais interatividade e capacidade adaptativa às características cognitivas e progresso do aluno. A localização e a recuperação de OA exigem que eles tenham sido cadastrados a partir de um modelo robusto de metadados, capaz de descrevê-los de forma completa e correta.

A extração do perfil e contexto do aluno é uma etapa fundamental nos SR [Pereira et al, 2018]. O perfil do aluno é uma representação do que se acredita saber sobre o conhecimento já adquirido, o progresso no conteúdo, o estilo preferido de mídia e outros tipos de informação. Os objetivos da aprendizagem, motivação, crenças e características pessoais estão muitas vezes associadas ao contexto educacional, bem como aos recursos tecnológicos utilizados. Este contexto é dinâmico e necessita de mecanismos para potencializar o reuso dos processos educacionais. O estilo de aprendizagem é refletido nas ações realizadas pelo aluno a partir dos estímulos recebidos do ambiente em que está interagindo. 
VIII Congresso Brasileiro de Informática na Educação (CBIE 2019)

Anais do XXX Simpósio Brasileiro de Informática na Educação (SBIE 2019)

No projeto BROAD a recomendação de recursos educacionais personalizados é feita pela aderência dos mesmos ao perfil e contexto do aluno ou grupos de alunos [Pereira et al, 2018] [Almeida et al., 2016] [Rezende et al. 2018] [Abdalla et al., 2017]. Para tal, tem-se recomendado recursos disponíveis em repositórios locais e em dados ligados, em ambientes virtuais de aprendizagem e nas redes sociais. O professor tem papel central no processo de recomendação, pois parte dele a seleção para a composição da seqüência do conteúdo a ser disponibilizada na disciplina para os alunos.

Analisar a utilização de uma sequiência de objetos por um aluno exige a identificação das escolhas feitas pelo mesmo. A proveniência dos dados [Buneman et al., 2001] nos fornece a documentação histórica do objeto e da sequiência dos objetos utilizados pelo aluno. Originalmente, os objetos de aprendizagem, através dos metadados, nos fornecem informações sobre a sua criação. A captura da proveniência do OA pode auxiliar na avaliação da qualidade em uso desse objeto, incluindo atributos como: adequação dos métodos e estratégias educacionais, atualização do conteúdo, e conformidade da mídia para apresentação do conteúdo.

A proposta é uma evolução do modelo de recomendação utilizado no projeto BROAD [Rezende et al. 2018], de forma a incluir também questões relacionadas à proveniência dos objetos de aprendizagem. Considerando a complexidade dos Sistemas de Recomendação Educacionais e a necessidade de sua assertividade e aderência aos interesses do aluno e objetivos educacionais a serem alcançados, o modelo proposto se baseia nos metadados capturados no processo de interação do aluno com os objetos de aprendizagem previamente selecionados pelo professor.

A questão de pesquisa que buscamos responder é: se definirmos uma arquitetura para modelar a proveniência prospectiva e capturar a proveniência retrospectiva dos objetos de aprendizagem selecionados pelo professor para uma certa disciplina, a mesma contribuirá para a assertividade das recomendações dos recursos educacionais? A solução proposta é a arquitetura BROAD-ProvCapture de captura de rastreabilidade da sequiência de OA executada pelo aluno (proveniência retrospectiva do $\mathrm{OA}$ ), associando as estratégias de ensino definidas pelo professor e estilos de aprendizagem do aluno, definidos no perfil explícito e na seqüência de uso desses OA.

O artigo está assim organizado: a seção 2 apresenta uma breve revisão da base teórica da proposta. A seção 3 discute os trabalhos relacionados. A seção 4 apresenta o BROAD-ProvCapture e sua avaliação. Na seção 5 estão as considerações finais.

\section{Pressupostos teóricos}

\subsection{Proveniência de Dados}

Proveniência de dados é a descrição do histórico de um dado desde sua criação até sua gravação em um banco de dados, bem como os processos que o influenciaram [Buneman et al., 2001]. É importante principalmente quando há a necessidade de entender o que o influenciou, como, e qual sua origem. Podemos dividir os tipos de proveniência em dois tipos: retrospectiva e prospectiva. A proveniência retrospectiva trata dos processos que influenciaram um dado até o presente momento, e a prospectiva trata dos modelos utilizados para se gerar a proveniência.

A proveniência de dados engloba diferentes domínios e aplicações, e cada uma tem seus requisitos. É necessário, portanto, definir os conteúdos, estruturas e atributos 
VIII Congresso Brasileiro de Informática na Educação (CBIE 2019)

Anais do XXX Simpósio Brasileiro de Informática na Educação (SBIE 2019)

que comporão as informações a serem capturadas. O modelo PROV [Belhajjme et al., 2012] é uma família de especificações proposta para expressar a proveniência de registros, contendo descrições de entidades e atividades envolvidas em produzir, entregar ou enunciar um determinado objeto. Como os dados de proveniência podem ter diferentes perspectivas e finalidades, o modelo PROV procura acomodar todos os tipos de uso da proveniência, e classifica a proveniência em três tipos:

- Centralizado em Agentes: os dados de proveniência visam especificar/registrar quem executou certas ações ou gerou certos objetos.

- Centralizada em Objetos: os dados de proveniência visam especificar quais são as partes que compõem um objeto.

- Centralizada em Processos: os dados de proveniência visam especificar como foram geradas as informações em questão.

O modelo PROV utiliza um grafo para representar informações de proveniência, caracterizado por três tipos de vértices [Groth and Moreau, 2013]: Entidade (algo físico, digital, conceitual ou outro tipo de coisa com alguns aspectos fixos, podendo as entidades ser reais ou imaginárias); Atividade (algo que ocorre durante um período de tempo, atuando sobre/com entidades. Assim, atividades podem consumir, processar, modificar, realocar ou gerar entidades); e Agentes (algo que possui certa responsabilidade por uma atividade, pela existência de uma entidade ou pelas atividades de outro agente).

No modelo PROV, existem também diversas relações de causalidade entre elas: used; wasGeneratedBy; wasAssociatedWith; wasAttributedTo; actedOnBehalfOf; wasRevisionOf; wasDerivedFrom; wasInformedBy; wasStartedBy e wasEndedBy.

Devido à importância das informações temporais em alguns cenários o modelo PROV oferece a possibilidade de guardar dados relativos ao tempo das informações de proveniência: data e hora relativas a entidades ou atividades, informações de geração ou uso de entidades e informações de início e término de execução para atividades.

A proveniência é representada por metadados [Groth and Moreau, 2013], porém, nem todos os metadados são necessariamente dados de proveniência. Nesse contexto, como as informações de proveniência podem ser obtidas de sistemas heterogêneos, representações diferentes e usadas por múltiplas aplicações, a interoperabilidade, que é sempre um requisito de um objeto de aprendizagem, é uma característica fundamental. Desta forma, o uso de proveniência neste contexto de sistemas de recomendação abre perspectivas de maior personalização.

\subsection{Perfil do aluno e estilos de aprendizagem}

Um aprendizado maior pode ocorrer quando estratégias de ensino combinam estilos de aprendizagem. Identificar estilos de aprendizagem não é rotular os alunos e adaptar as instruções para adequá-las às suas preferências, mas propor modelos de recomendação que sejam cada vez mais aderentes ao seu perfil e contexto. Nesse cenário a captura de rastreabilidade do projeto dos OA e da orquestração dos alunos no seu uso pode trazer contribuições. A busca pela personalização das recomendações de objetos de aprendizagem aderentes ao perfil e contexto do aluno e de grupos de alunos apresenta desafios às pesquisas da área de Sistemas de Recomendação Educacionais.

Existem diversos modelos que descrevem classificações de um aluno e em um determinado estilo de aprendizagem [Zaina and Bresnann, 2012] [Buiar, et al., 2017] 
VIII Congresso Brasileiro de Informática na Educação (CBIE 2019)

Anais do XXX Simpósio Brasileiro de Informática na Educação (SBIE 2019)

[Silva et al., 2016]. "Na personalização de recursos educacionais, algumas características do aluno são relevantes, como o perfil do estudante, que pode ser caracterizado por meio do emprego do estilo de aprendizagem que determina como o aluno interage com o sistema de e-learning, suas preferências, o que torna possível ao sistema adaptativo fornecer conteúdos relevantes para o aluno [Zaina et al. 2012]."

Nascimento et al. (2017) mostraram como resultado de uma Revisão Sistemática da Literatura, que o modelo de estilo de aprendizagem mais utilizado e citado é o de Felder-Silverman. Este modelo destaca o emprego do LOM (Learning Object Metadata), como padrão de metadados e a especificação e o gerenciamento de metadados empregados como mecanismo de apoio para recomendação. Além disso, propõe quatro dimensões [Buiar et al. 2017] que serão adotadas na solução proposta.

\section{Trabalhos relacionados}

Dietze et al.(2013) apresentam o Modelo de Gerenciamento de Recursos e Metadados (ReM3). O modelo de informações baseia-se na arquitetura e nos padrões da Web, mais especificamente nos princípios do Linked Data ao gerenciar recursos junto com seus metadados. Permite expressar relações entre metadados e acompanhar a procedência e o controle de acesso. O ReM3 suporta a proveniência centrada no agente, o que significa que ele acompanha as informações sobre quais usuários estavam envolvidos na criação ou modificação de informações e a proveniência centrada no objeto, ou seja, rastrear as origens de um recurso ou seus metadados.

Coccoli \& Torre (2014) abordam a web semântica e educação para melhoria da aprendizagem. Propõem o modelo SWOT que é responsável por gerenciar conteúdos. Utilizam proveniência de dados para obter perfil do aluno e assim fazer recomendações apropriadas para cada tipo de aluno conforme seu nível de aprendizagem. Todos os recursos estão voltados para dados ligados.

Destacamos em rastreabilidade de objetos de aprendizagem a pesquisa de [Celino and Dell'Aglio, 2011] sobre Rastreamento de Proveniência no aprendizado de simulação. Os autores definem a rastreabilidade em dois níveis: (i) no projeto - quando os cenários de aprendizagem são modelados na base de conhecimento com suas possíveis regras de planejamento, e (ii) nas sessões de aprendizagem - quando os resultados das simulações são analisados. No projeto, a proveniência pode ser usada para rastrear as cadeias de causa-efeito entre os possíveis eventos de simulação. As regras de conclusão e inferência podem ser exploradas para a verificação de consistência da modelagem da simulação. Após as sessões de aprendizagem, os registros de simulação podem ser analisados para entender e sintetizar os resultados da aprendizagem como as necessidades de aprendizagem, comportamentos comuns, procedimentos, entre outros.

Em termos de categorização dos modelos e estilos de aprendizagem e os cenários que cada um deles melhor se aplica, tem-se o trabalho de [Carvalho et. al., 2017] que buscam melhorar os sistemas adaptativos para a educação usando uma ontologia sobre Objetos de Aprendizagem e os estilos dos alunos, usando o modelo de Felder-Silverman.

Labib et al. (2017) apresentam uma ontologia de modelos de aprendizagem para melhorar o ambiente de ensino dos estudantes. Neste artigo os autores classificam 
VIII Congresso Brasileiro de Informática na Educação (CBIE 2019)

Anais do XXX Simpósio Brasileiro de Informática na Educação (SBIE 2019)

os modelos e estilos e cruzam informações dos estilos de aprendizagem de forma a recomendar o conteúdo mais aderente ao perfil do aluno.

O trabalho de Dietze et al. (2013) assim como o BROAD-ProvCapture utilizam proveniência de dados para obter informações em relação aos metadados, o que reforça o uso de proveniência retrospectiva. Assim como o BROAD-ProvCapture [Coccoli \& Torre, 2014] abrangem também o contexto de SR. A representação de modelos e estilos de aprendizagem [Carvalho et. al., 2017] e [Labib et al., 2017] em ontologias, permitem automatizar o processo de identificação do perfil do aluno, conforme proposto na arquitetura BROAD-ProvCapture. A proposta neste trabalho é observar o caminho do aluno enquanto usa uma seqüência de OA, pré-definida pelo professor, através de uma arquitetura capaz de adotar técnicas e mecanismos para capturar suas preferências. Para tal, identificar as características marcantes de um determinado aluno, associando suas preferências aos estilos de aprendizagem, auxilia nas recomendações de OA que influenciam seu processo de aprendizagem.

\section{BROAD-ProCapture: uma arquitetura para proveniência de objetos de aprendizagem}

O uso de proveniência em seqüência de OA se justifica pelas seguintes características e possibilidades de aplicação: Confiabilidade da indicação pelo professor; Reprodutividade da recomendação para um conjunto de alunos com perfil semelhante; Definição de métodos e estratégias adequados para uso dos OA; Identificação da melhor forma de apresentação de conteúdos; Seleção de atividades e mídias direcionadas a cada estilo de aprendizagem; e Identificação de requisitos para desenvolvimento de aplicações educacionais semanticamente ricas.

A proposta é fornecer ao professor a identificação automática do estilo preferencial de aprendizagem do aluno, em uma dada situação de ensino. Nesta situação, o professor seleciona, previamente, um conjunto de objetos de aprendizagem para compor uma seqüência de um conteúdo programático.

O modelo PROV, voltado para questões de responsabilidades e histórico de dados, possui diversas relações entre os agentes e os demais tipos (atividades e entidades), e atende aos requisitos do projeto. Considerando ser a arquitetura proposta focada na captura de dados relacionados em grande parte aos metadados dos objetos de aprendizagem, entendemos ser esse modelo adequado. A figura 1 apresenta as duas etapas principais do processo de planejamento e uso das seqüências de objetos de aprendizagem, e a captura dos dados de proveniência.

A rastreabilidade na arquitetura proposta se dá em dois níveis:

- Prospectiva - obtida no nível de projeto de construção dos objetos de aprendizagem selecionados pelo professor, a partir de um plano didático da disciplina. Os itens serão capturados pelos metadados dos objetos de aprendizagem cadastrados.

- Retrospectiva - obtida a partir da captura de proveniência da navegação do aluno na sequiência selecionada para a sessão de estudo.

Considerando que na arquitetura o conteúdo da proveniência se dará pela captura das informações em diferentes etapas, os seguintes itens serão armazenados:

- Responsabilidades: itens relativos ao professor e ao planejamento da sessão de estudo (curso, disciplina, unidade de conteúdo, lição, programa, nível educacional, área de conhecimento, sequiencia do conteúdo, estratégias pedagógicas, objetivos educacionais e população alvo). 
VIII Congresso Brasileiro de Informática na Educação (CBIE 2019)

Anais do XXX Simpósio Brasileiro de Informática na Educação (SBIE 2019)

- Itens de metadados: na primeira versão serão considerados o nível de interatividade, o tipo de interatividade e o tipo de objeto de aprendizagem.

- Itens de preferência: captura a seqüência percorrida pelo aluno na sessão de estudo, com o objetivo de identificar os objetos de aprendizagem preferenciais.

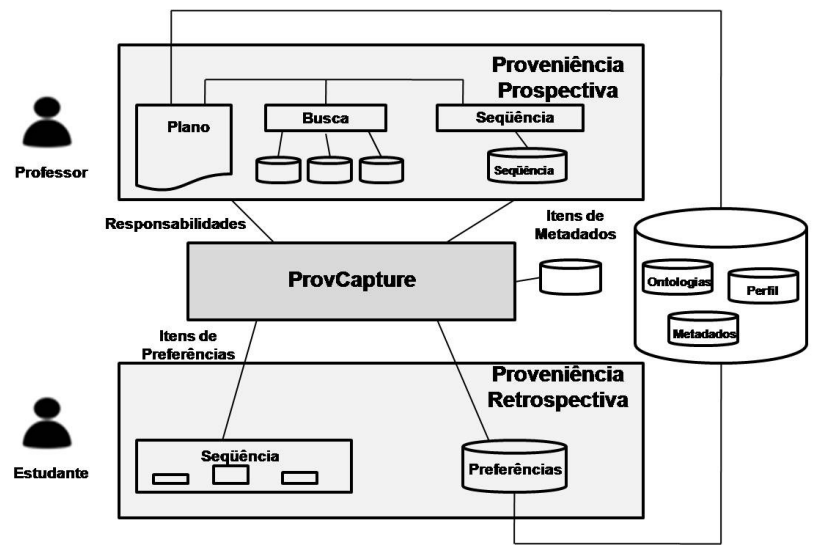

Figura 1 - Arquitetura BROAD-ProvCapture.

$\mathrm{Na}$ arquitetura a base de conhecimento é representada pelas ontologias, pelos itens de metadados armazenados e pelos perfis dos alunos. Essa camada apóia a proposta em diferentes etapas: Planejamento (elaboração do plano, busca e seleção de OA, seqüência de OA) e execução (seqüência de OA e identificação de preferências).

Para a identificação dos estilos de aprendizagem do aluno através da captura das escolhas e uso dos objetos de aprendizagem recomendados pelos professores, o relacionamento entre o padrão de metadados LOM e o perfil do usuário foi construído. Para tanto, foi utilizada a categoria de metadados educacionais.

O Nível de Interatividade refere-se ao grau de interatividade que caracteriza o objeto de aprendizagem. Refere-se ao grau em que o aluno pode influenciar os aspectos ou comportamentos do objeto de aprendizagem. Os que possuem o valor "ativo" para o campo tipo de interatividade podem ter um alto nível de interatividade (por exemplo, um ambiente de simulação com vários controles) ou um nível de interatividade baixo (por exemplo, um conjunto de instruções que solicitem uma atividade). Já os objetos que possuem o valor "expositivo" podem ter um nível de interatividade baixo (por exemplo, um texto narrativo linear) ou um nível de médio a alto (por exemplo, um documento de hipertexto com diversos links).

O campo Tipo de Interatividade diz respeito ao modo predominante de aprendizagem suportado pelo objeto de aprendizagem. Pode possuir os valores ativo e expositivo. O valor ativo, ligado a uma aprendizagem "ativa" (também conhecido como "aprender fazendo") é apoiado por conteúdo que induz a uma ação produtiva diretamente pelo aluno. Um OA ativo pede ao aluno uma entrada semanticamente significativa e incluem simulações, jogos, questionários e exercícios. Aprendizagem "expositiva" (também conhecida como "aprendizagem passiva") ocorre quando o trabalho do aluno consiste principalmente em absorver o conteúdo exposto a ele (geralmente através de textos, imagens ou sons). No caso de um objeto de aprendizagem combinar os tipos de interatividade ativo e expositivo, o seu tipo é considerado misto.

O campo Tipo de objeto de aprendizagem especifica um conjunto de objetos definidos como: Simulação, Exercício, Resolução de Problemas, Jogo, Vídeo, Hipertexto, Áudio, Imagem, Slide, Texto e Hipermídia, entre outros. 
VIII Congresso Brasileiro de Informática na Educação (CBIE 2019)

Anais do XXX Simpósio Brasileiro de Informática na Educação (SBIE 2019)

Durante a navegação em uma seqüência de OA é capturado o caminho selecionado pelo aluno (proveniência retrospectiva) e extraídos itens que, através de regras semânticas (ou ontológicas), identificam seu estilo de aprendizagem. Conforme especificado anteriormente, para fins deste trabalho adotamos o modelo de FelderSilverman. O estilo de aprendizagem reflete as preferências do aluno e facilita a recomendação dos objetos aderentes a um dos perfis. O Quadro 1 destaca algumas relações entre metadados LOM utilizados e as estratégias e Estilos de Aprendizagem que compõem as dimensões adotadas para identificação das estratégias de ensino a serem recomendadas como resultado dos estilos de aprendizagem identificados.

\section{Quadro 1 - Relação entre tipos de OA, tipo e nível de interatividade, estilo de} aprendizagem e estratégia.

\begin{tabular}{|l|l|l|l|l|}
\hline \multicolumn{1}{|c|}{ Tipo } & \multicolumn{1}{c|}{$\begin{array}{c}\text { Tipo de } \\
\text { Interatividade }\end{array}$} & $\begin{array}{c}\text { Nível de } \\
\text { Interatividade }\end{array}$ & \multicolumn{1}{c|}{$\begin{array}{c}\text { Estilo de } \\
\text { Aprendizagem }\end{array}$} & \multicolumn{1}{c|}{ Estratégia } \\
\hline $\begin{array}{l}\text { Simulação/Exercício/ } \\
\begin{array}{l}\text { Resolução de } \\
\text { problemas/Jogo }\end{array}\end{array}$ & Ativo & $\begin{array}{l}\text { Alto } \\
\text { Muito alto }\end{array}$ & $\begin{array}{l}\text { Intuitivo } \\
\text { Ativo } \\
\text { Auditivo/Visual }\end{array}$ & $\begin{array}{l}\text { Ativa } \\
\text { Abstrata } \\
\text { Visual/Verbal }\end{array}$ \\
\hline $\begin{array}{l}\text { Vídeo/Hipertexto/Áudio } \\
\text { /Imagem/Slide/Texto }\end{array}$ & Expositivo & $\begin{array}{l}\text { Baixo } \\
\text { Muito baixo }\end{array}$ & $\begin{array}{l}\text { Sensorial } \\
\text { Reflexivo } \\
\text { Auditivo/Visual }\end{array}$ & $\begin{array}{l}\text { Passiva } \\
\text { Concreta } \\
\text { Visual/Verbal }\end{array}$ \\
\hline Hipermídia & Misto & Médio & $\begin{array}{l}\text { Intuitivo/Sensorial } \\
\text { Ativo/Reflexivo } \\
\text { Auditivo/Visual }\end{array}$ & $\begin{array}{l}\text { Ativa/Passiva } \\
\text { Abstrata/Concreta } \\
\text { Visual/Verbal }\end{array}$ \\
\hline
\end{tabular}

A idéia é que a partir da execução da seqüência de objetos de aprendizagem pelo aluno seja identificado seu perfil e ressaltadas suas preferências, de forma que auxiliem na sugestão posterior de novas seqüências para aquele aluno ou grupos de alunos com perfis convergentes. Para tal, foram criadas algumas regras, tais como:

tipo interatividade ativo $\wedge$ nível interatividade alto $\vee$ muito alto $=>$ estilo de aprendizagem intuitivo $\wedge$ ativo $\wedge$ auditivo $\checkmark$ visual

tipo interatividade expositivo $\wedge$ nível interatividade baixo $\vee$ muito baixo $=>$ estilo de aprendizagem sensorial $\wedge$ reflexivo $\wedge$ auditivo $\vee$ visual

tipo interatividade misto $\wedge$ nível interatividademédio $\Rightarrow>$ estilo de aprendizagem intuitivo $\wedge$ ativo $\wedge$ sensorial $\wedge$ reflexivo $\wedge$ auditivo $\vee$ visual

estilo de aprendizagem intuitivo $\wedge$ ativo $\wedge$ auditivo $\vee$ visual $=>$ estratégia ativa $\wedge$ abstrata $\wedge$ visual $\vee$ verbal estilo de aprendizagem sensorial $\wedge$ reflexivo $\wedge$ auditivo $\vee$ visual $=>$ estratégia passiva $\wedge$ concreta $\wedge$ visual $\vee$ verbal.

As regras foram usadas para inferir o nível de aderência do aluno a um determinado tipo de perfil e a indicação das estratégias equivalentes, em três níveis:

High $\geq 75 \%$ dos objetos executados atendem a um estilo de aprendizagem - totalmente aderente ao perfil Mid $\geq 50 \%$ dos objetos executados atendem a um estilo de aprendizagem - aderente ao perfil Low $<50 \%$ dos objetos executados atendem a um estilo de aprendizagem - perfil não definido.

\subsection{Estudo de viabilidade}

Para avaliação da arquitetura BROAD-ProvCapture foi realizado um estudo de viabilidade, com a formalização do estudo de caso baseada em [Drescht et al., 2015], a fim de contribuir para a avaliação da questão de pesquisa formulada e verificação do artefato desenvolvido. A questão de pesquisa que buscamos responder é: se definirmos uma arquitetura de captura de proveniência, para além de detectar os metadados dos objetos de aprendizagem selecionados pelo professor para a sessão de estudo, definidos 
VIII Congresso Brasileiro de Informática na Educação (CBIE 2019)

Anais do XXX Simpósio Brasileiro de Informática na Educação (SBIE 2019)

como uma seqüência recomendada a ser seguida, a mesma será capaz de capturar as informações sobre a navegação dessa seqüência pelo aluno, indicando assertividade da sequiência? A avaliação seguiu as seguintes etapas: definição, formulação do objetivo, planejamento, execução e observação das evidências e apresentação dos resultados.

$\mathrm{O}$ estudo foi realizado num ambiente real de aprendizagem, na Universidade Federal de Juiz de Fora, na disciplina Fundamentos de Sistemas de Informação. Essa disciplina compõe o Núcleo Básico das grades curriculares da área de Ciência da Computação. Foi utilizado o Ambiente Virtual Moodle e a BROAD-ProvCapture foi integrada como serviço. A Figura 2 apresenta o conjunto de objetos selecionados e indicados pelo professor, previamente cadastrados com os metadados LOM, para uma sessão de estudo. A turma era composta de 78 alunos.

Foi disponibilizada para os alunos uma seqüência de OA de diferentes tipos, níveis de interação e tipos de interação. Para esse estudo, o professor selecionou um documento PDF, uma apresentação, uma vídeoaula e um jogo (Figura 2). Os quatro recursos disponibilizados para os alunos podem ser classificados como (Quadro 2).

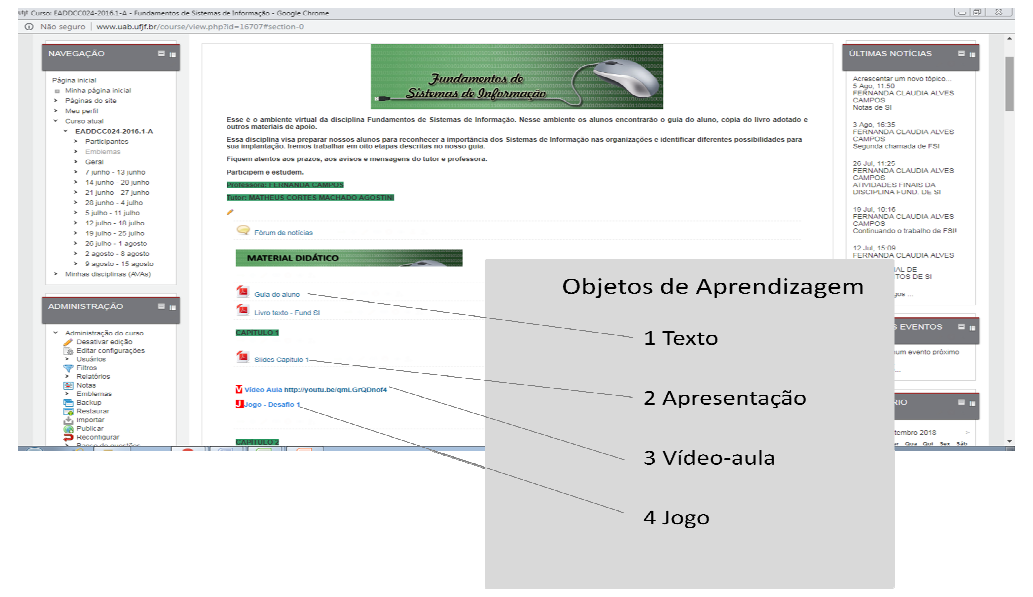

Figura 2 - Ambiente da disciplina com os OA selecionados em uma sessão de estudo.

Quadro 2 - Classificação dos Objetos de Aprendizagem disponibilizados.

\begin{tabular}{|l|l|l|l|l|}
\hline \multicolumn{1}{|c|}{ Tipo } & $\begin{array}{c}\text { Tipo de } \\
\text { Interatividade }\end{array}$ & $\begin{array}{c}\text { Nível de } \\
\text { Interatividade }\end{array}$ & \multicolumn{1}{c|}{$\begin{array}{c}\text { Estilo de } \\
\text { Aprendizagem }\end{array}$} & \multicolumn{1}{c|}{ Estratégia } \\
\hline $\begin{array}{l}1 \text { Texto } \\
2 \text { Slides } \\
3 \text { Vídeo }\end{array}$ & Expositivo & $\begin{array}{l}\text { Baixo } \\
\text { Muito baixo }\end{array}$ & $\begin{array}{l}\text { Sensorial } \\
\text { Reflexivo } \\
\text { Auditivo/Visual }\end{array}$ & $\begin{array}{l}\text { Passiva } \\
\text { Concreta } \\
\text { Visual/Verbal }\end{array}$ \\
\hline 4 Jogo & Ativo & $\begin{array}{l}\text { Alto } \\
\text { Muito alto }\end{array}$ & $\begin{array}{l}\text { Intuitivo } \\
\text { Ativo } \\
\text { Auditivo/Visual }\end{array}$ & $\begin{array}{l}\text { Ativa } \\
\text { Abstrata } \\
\text { Visual/Verbal }\end{array}$ \\
\hline
\end{tabular}

Essa seqüência, apesar de apresentada na forma de lista, permite a livre escolha dos alunos. A navegação de cada aluno pode indicar a sua preferência que retrata o seu estilo de aprendizagem. Pelas opções ofertadas temos várias possibilidades de fluxo de navegação, a saber:

1. Fluxos com predominância do tipo de interatividade expositiva e nível de interatividade baixo, indicam que as estratégias de ensino mais aderentes ao perfil do aluno são do tipo passivas, como aulas expositivas: $1,2,3$, e, 4; 2,1, 3, e, 4; e 1, 3, 2, e, 4 . 
VIII Congresso Brasileiro de Informática na Educação (CBIE 2019)

Anais do XXX Simpósio Brasileiro de Informática na Educação (SBIE 2019)

2. Fluxos com predominância do tipo de interatividade ativo e nível de interatividade alto, indicam que as estratégias de ensino mais aderentes ao perfil do aluno são do tipo ativas, como desafios, projetos, entre outras: 4, 2, 3, e, 1; e 4, 3, 1 e 2 .

Ao ser capturada a sequiência da sessão de estudo de um aluno, o fluxo das escolhas permite, através de regras ontológicas da base de conhecimento, inferir características desse aluno. Entretanto, as diferentes escolhas da seqüência permitiram identificar os métodos e as estratégias prioritárias dos alunos, as preferências dos tipos de objetos e principalmente dar ao professor feedback sobre o uso dos OA. Esse perfil capturado implicitamente dos alunos é incorporado ao sistema de recomendação.

Os ambientes virtuais de aprendizagem, como o Moodle, adotado nesse estudo, em geral, possuem ferramentas que indicam o fluxo de acesso dos alunos, entretanto, esse relatório não retrata a navegação nos objetos de aprendizagem e sim em todas as opções de interação como fóruns, tarefas, etc. A vantagem de se adotar um modelo de proveniência é o rastro que se pode armazenar de todas as sessões de estudo do aluno. As evidências observadas não podem ainda ser generalizadas, mas a proveniência de dados, que hoje é empregada em diferentes domínios, pode ser aplicada em sistemas de recomendação e contribuir para o desenvolvimento de aplicações educacionais mais aderentes às características dos alunos.

\section{Considerações finais}

Ao capturarmos a proveniência dos OAs buscamos identificar características de aprendizagem e orientações de estudo que contemplem os diferentes estilos de aprendizagem nas recomendações aderentes ao perfil e contexto do aluno. Nessa pesquisa integramos a proveniência prospectiva e a retrospectiva visando abordar aspectos educacionais e pessoais como parte de um modelo de perfil do aluno. Para o perfil e contexto do aluno são necessárias a definição e a inclusão de mais itens, porém sendo um trabalho em andamento as restrições não inviabilizaram as avaliações iniciais. Podem ainda ser capturadas de forma implícita ou explícita: motivação, interesse, importância do objeto, utilidade para o seu processo de aprendizagem, entre outros.

Em termos de navegação identificamos que alguns alunos reproduzem a seqüência sugerida pelo professor não se esforçando ou permitindo inovação. Outros questionam a orientação e adotam outras abordagens de navegação e exploram os novos materiais. É esse rastro da navegação que nos permite armazenar informações sobre as suas preferências. A proposta descrita neste artigo possui limitações em relação à generalização dos resultados, uma vez que a total implementação da solução está sendo feita de forma evolutiva e o estudo experimental foi feito em uma disciplina. Todavia, este serviu para indiciar possibilidades de melhorar a proposta da arquitetura e sua aplicabilidade em sistemas de recomendação educacionais.

Agradecimentos: FAPEMIG, CNPq, UFJF e Capes pelo apoio financeiro. Aos professores e alunos que participaram da avaliação da proposta.

\section{Referências}

Abdalla, A., Ströele, V., Veiga, W., Simões, L., Campos, F., Braga, R., \& David, J. M. N. (2017). R . ECOS - Educational Recommender Ecosystem. In IEEE/ACM Joint 5th International Workshop on Software Engineering for Systems-of-Systems and 11th Workshop on Distributed Software Development, Software Ecosystems and Systems-ofSystems, p. 48-54. 
VIII Congresso Brasileiro de Informática na Educação (CBIE 2019)

Anais do XXX Simpósio Brasileiro de Informática na Educação (SBIE 2019)

Almeida, R. F., Pereira, C. K., Campos, F. e Ströele, V. (2016). Recomendação de Recursos Educacionais para Grupos: buscando soluções em Redes Sociais. In Anais do XXVII Simpósio Brasileiro de Informática na Educação - SBIE.

Belhajjame, K., B'Far, R., J. Cheney, S. Coppens, S. Cresswell, Y. Gil, P. Groth, G. Klyne, T. Lebo, J. McCusker, et al. (2013) Prov-dm: The prov data model. W3C Recommendation.

Buiar, J. A., P., Andrey R., Oliveira, L. E. S. (2017) Identificação de Estilo de Aprendizagem: Um modelo de inferência automatizado baseado no perfil de personalidade identificado nos textos produzidos pelo aluno. Anais do XXVIII Simpósio Brasileiro de Informática na Educação - SBIE.

Buneman, P., Khanna, S., Tan, W.C., (2001) Why and where: A characterization of data provenance. In: 8th International Conference on Database Theory, London. p. 4-6.

Carvalho, Victor de C. de et alli. (2017) OntAES: Uma Ontologia para Sistemas Adaptativos Educacionais Baseada em Objetos de Aprendizagem e Estilos de Aprendizagem. Anais do XXVIII Simpósio Brasileiro de Informática na Educação - SBIE. 1307-1316.

Coccoli, M., and Torre, I.. (2014) Interacting with annotated objects in a semantic web of things application. Journal of Visual Languages \& Computing, 25(6):1012-1020.

Dietze, S., Taibi, D., Yu, H. Q. and Dovrolis, N.. (2015) Al inked d ataset of medical educational resources. British Journal of Educational Technology, 46(5):1123-1129.

Drescht, A.; Lacerda, D.P.; Antunes Junior, J.A.V. (2015). Design Science Research: método para avanço da ciência e tecnologia. Porto Alegre, Bookman, p 204.

Groth, P., Moreau, L., (2013), PROV- Overview: An Overview of the PROV Family of Documents. Available at: <https://www.w3.org/TR/2013/NOTE-prov-overview20130430/>. Accessed on: Jan. 2018.

Labib, A. Ezzat, José H. Canós, and M. Carmen Penadés. On the way to learning style models integration: a Learner's Characteristics Ontology. Computers in Human Behavior, p. 433445.

Nascimento, P., Barreto, R., Primo, T., Gusmão, T., Oliveira, E. (2017). Recomendação de Objetos de Aprendizagem baseada em Modelos de Estilos de Aprendizagem: Uma Revisão Sistemática da Literatura. In Simpósio Brasileiro de Informática na Educação-SBIE .Vol. 28, No. 1, p. 213.

Pereira, C. K., Campos, F., Ströele, V., David, J. M. N. Braga, R. (2018). BROAD-RSI educational recommender system using social networks interactions and linked data. Journal of Internet Services and Applications, v. 9, n. 1, p 7.

Rezende, P. A., Campos, F., Stroele, V., Braga, R., \& David, J. M. N. (2018) Recomendação Baseada no Perfil e Contexto Tecnológico do Aluno. In Simpósio Brasileiro de Informática na Educação-SBIE. p. 1273-1282.

Silva, Z. C., Ferreira, L.R., Pimentel, A.R. (2016) Modelo de Apresentação Adaptativa de Objeto de Aprendizagem baseada em Estilos de Aprendizagem. In: V Congresso Brasileiro de Informática na Educação - CBIE, pp. 217-726, 2016.

Valaski, J., Malucelli, A., Reinehr, S. (2011) Revisão dos Modelos de Estilos de Aprendizagem Aplicados à Adaptação e Personalização dos Materiais de Aprendizagem. In: XXII Simpósio Brasileiro de Informática na Educação - SBIE, Aracaju, pp. 844-847.

Zaina, L. A. M, (2012) e-LORS: Uma Abordagem para Recomendação de Objetos de Aprendizagem. Revista Brasileira de Informática na Educação, Volume 20, Número 1. 4-14. 